\title{
Association of miR-34a, miR-130a, miR-150 and miR-155 polymorphisms with the risk of ischemic stroke
}

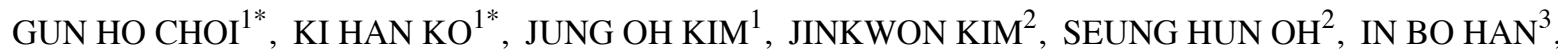 \\ KYUNG GI CHO ${ }^{3}$, OK JOON KIM ${ }^{2}$, JINKUN BAE ${ }^{4}$ and NAM KEUN KIM ${ }^{1}$ \\ ${ }^{1}$ Department of Biomedical Science, College of Life Science, CHA University, Seongnam 463-400; \\ Departments of ${ }^{2}$ Neurology, ${ }^{3}$ Neurosurgery and ${ }^{4}$ Emergency Medicine, CHA Bundang Medical Center, \\ School of Medicine, CHA University, Seongnam 463-712, Republic of Korea
}

Received October 21, 2015; Accepted May 11, 2016

DOI: $10.3892 /$ ijmm.2016.2609

\begin{abstract}
MicroRNAs (miRNAs or miRs) are small (19-23 nt) non-coding RNA molecules that are endogenous regulators of gene expression. Previous studies have found that some miRNAs are related to the progression of ischemia in the cerebral artery. Furthermore, a recent study found a significant association between miRNA single nucleotide polymorphisms (SNPs) and the risk of ischemic stroke. Therefore, it may be valuable to investigate associations between megakaryocyte formationrelated miRNA polymorphisms and the prevalence of ischemic stroke. We thus conducted a case-control study of 1,000 individuals who were screened for 4 miRNA polymorphisms (miR-34a rs6577555C >A, miR-130a rs731384C >T, miR-150 rs73056059G $>\mathrm{A}$ and $m i R-155$ rs767649T $>\mathrm{A}$ ) by PCR-RFLP analysis. The study population comprised 596 patients with ischemic stroke and 404 control subjects without any history of neurological disorders. We observed associations between miRNA polymorphisms and individual stroke subtypes. The miR-150 polymorphisms were significantly associated with ischemic stroke subgroups, such as left anterior descending artery (LAD) disease [GG vs. AA: adjusted odds ratio (AOR), 1.922; 95\% confidence interval (CI), 1.003-3.681] and cardioembolism (GG vs. AA: AOR, 2.996; 95\% CI, 1.2936.939). Additionally, Cox proportional analysis indicated that the $m i R$-150GA genotype was associated with survival in patients with ischemic stroke [adjusted hazard ratio (HR), 2.063; 95\% CI,
\end{abstract}

Correspondence to: Professor Nam Keun Kim, Department of Biomedical Science, College of Life Science, CHA University, 335 Pangyo-ro, Bundang-gu, Seongnam 463-400, Republic of Korea E-mail: nkkim@cha.ac.kr

Professor Jinkun Bae, Department of Emergency Medicine, CHA Bundang Medical Center, School of Medicine, CHA University, 351 Yatap-dong, Bundang-gu, Seongnam 463-712, Republic of Korea E-mail: galen97@naver.com

*Contributed equally

Key words: microRNA, ischemic stroke, polymorphisms, $m i R-34 a$, miR-130a, miR-150, miR-155
1.142-3.727; $\mathrm{P}=0.017$ ] and with the LAD subgroup [adjusted HR, $3.021 ; 95 \%$ CI, 1.345-6.785; $\mathrm{P}=0.008]$. Our findings suggest that miR-150 polymorphisms may contribute to the development of ischemic stroke and may potentially act as biomarkers to predict the risk of ischemic stroke. To the best of our knowledge, this is the first study to evaluate the association between miRNA polymorphisms ( $m i R-34 a \mathrm{C}>\mathrm{A}, m i R-130 a \mathrm{C}>\mathrm{T}, m i R-150 \mathrm{G}>\mathrm{A}$ and $m i R-155 \mathrm{~T}>\mathrm{A})$ and ischemic stroke.

\section{Introduction}

Stroke is the second most common cause of mortality worldwide (1), and approximately $84 \%$ of these deaths are caused by ischemic stroke (2). In South Korea, stroke is the second most frequent cause of death after cancer and is more prevalent than heart disease. Stroke has well-known risk factors, including hypertension, diabetes mellitus, advanced age, smoking, hyperlipidemia, hyperhomocysteinemia and a thrombophilia event in blood vessels $(1,2)$. A number of different diseases can cause ischemic stroke. The most common source of ischemic stroke is arterial occlusion in the head, usually caused by atherosclerosis, gradual cholesterol deposition or thrombosis $(3,4)$. Blood vessel occlusion is primarily caused by thrombosis $(53 \%$ of cases) or embolism (31\% of cases) (5). Thrombus formation can be caused by excessive platelet generation (6), and the association between platelets and ischemic stroke has been widely studied (7-9). Platelets are involved in both normal hemostasis and thrombosis (10). They are formed in the cytoplasm of megakaryocytes (MKs), their precursor cells, located in the bone marrow (11).

MicroRNAs (miRNAs or miRs) are a class of small (19-23 nt in length), endogenous non-coding RNA molecules that are endogenous physiological regulators of gene expression (12). miRNAs efficiently control gene expression by binding 3'-untranslated regions in mRNAs to downregulate their protein expression (13). miRNAs play important roles in a number of physiological and pathological processes, including metabolism (14), hematopoiesis (15) and immune function (16). Moreover, previous studies have indicated that temporal miRNA regulation may be related to the progression of ischemia in the cerebral artery $(17,18)$. Furthermore, a recent study found a significant association between miRNA single nucleotide poly- 
morphisms (SNPs) and the risk of ischemic stroke (19). As has been previously reported by Edelstein et al (20), as regards the regulated function of megakaryocytopoiesis, various miRNAs play a crucial role in this process, as well as in platelet biogenesis. Therefore, it may be valuable to investigate associations between MK formation-related miRNAs, such as miR-34a, miR-130a, $m i R-150$ and $m i R-155(20,21)$, and the prevalence of ischemic stroke.

In this study, we investigated the associations between 4 miRNA polymorphisms ( $m i R-34 a$ rs6577555C $>$ A, $m i R-130 a$ rs731384C $>\mathrm{T}, m i R-150$ rs73056059G $>\mathrm{A}$ and $m i R-155$ rs767649T $>A$ ) and the risk of ischemic stroke in a Korean population.

\section{Subjects and methods}

Subjects. The study population comprised 596 patients with ischemic stroke (mean age \pm SD: 63.67 \pm 10.42 years, 254 males, 342 females). Ischemic stroke was diagnosed based on rapidly developing neurological symptoms with a concurrent acute infarction, documented by brain magnetic resonance imaging (MRI). In addition, 404 control subjects were included (mean age \pm SD: $63.66 \pm 10.47$ years, 173 males, 231 females). The patients were enrolled by consecutive referral between July 1, 2000 and February 28, 2008 in the Neurology Department at CHA Bundang Medical Center (Seongnam, South Korea). The control subjects were recruited from patients who visited the hospital for a routine health examination. The exclusion criteria for the control sujbects were as follows: a family history of stroke or experiencing non-specific dizziness, non-organic headaches, or anxiety during the enrollment period. All control subjects underwent some form of brain imaging ( $75 \% \mathrm{MRI})$, and no organic cerebral lesions were observed. The subjects were interviewed to collect clinical information regarding demographic data and vascular risk factors. Subjects with a previous history of cerebral hemorrhage or those with incomplete medical histories were excluded from this study. The patients with ischemic stroke were classified into 3 subgroups as follows: 202 patients had left anterior descending artery (LAD) disease, 143 had small-vessel disease (SVD) and 57 had cardioembolism (CE); 194 patients had an undetermined etiology.

Ischemic stroke was defined as a stroke (characterized by rapidly developing clinical symptoms and signs of focal and/or global brain function loss) with evidence of a cerebral infarction in clinically relevant areas of the brain based on a brain MRI. Based on clinical manifestations and neuroimaging data, two neurologists classified ischemic stroke into 3 etiological subtypes using the criteria from the Trial of Org 10172 in Acute Stroke Treatment (TOAST) clinical trial as follows (22): i) subtype 1: LAD, an infarct lesion of $\geq 15 \mathrm{~mm}$ in diameter, as determined by an MRI, and significant ( $>50 \%$ ) stenosis of a major brain artery or a branch cortical artery, as determined by cerebral angiography, with symptoms associated with that arterial territory; ii) subtype 2: SVD, an infarct lesion of $<15 \mathrm{~mm}$, but $>5 \mathrm{~mm}$ in diameter, as determined by an MRI, and classic lacunar syndrome without evidence of cerebral cortical dysfunction or a potentially detectable cardiac source for the embolism; and iii) subtype 3: CE, arterial occlusions presumably due to a heart-originated embolus, as detected by cardiac evaluation. We measured clinical parameters, including hypertension, diabetes, hyperlipidemia, homocysteine levels, folate levels, vitamin B12 levels, cholesterol, platelet (PLT) count, PT, prothrombin time (PT), activated partial thromboplastin time (aPTT), fibrinogen levels, antithrombin levels, BUN levels and uric acid levels based on previously described methods $(23,24)$.

Genetic analysis. Genomic DNA was extracted from blood leukocytes using the G-DEX blood extraction kit (Intron Inc., Seongnam, Korea). The 4 most well-studied SNPs in the miRNAs were determined by a documentary search which included promoter region SNPs (miR-34a rs6577555C $>$ A, miR-130a rs731384C $>$ T, miR-150 rs73056059G $>$ A and miR-155 rs767649T $>A$ ). All SNP sequences were obtained from the HapMap database (www.hapmap.org) and $\operatorname{dbSNP} \approx(w w w$. ncbi.nlm.nih.gov/projects/SNP/). Nucleotide alterations were determined by polymerase chain reaction (PCR)-restriction fragment length polymorphism (RFLP) analyses using the isolated genomic DNA as a template. PCR for the $m i R-34 a$ rs6577555C $>\mathrm{A}$ polymorphism was performed using the following primers: 5'-CCT GGT TAA CAT AGC CAG AGC-3' (forward) and 5'-GCA GAC ATG CTG ACT TTT CAA-3' (reverse). DNA was amplified over 35 cycles (denaturation at $95^{\circ} \mathrm{C}$ for $30 \mathrm{sec}$, annealing at $56^{\circ} \mathrm{C}$ for $30 \mathrm{sec}$, and extension at $72^{\circ} \mathrm{C}$ for $\left.35 \mathrm{sec}\right)$. The PCR products were digested with the BanII restriction endonuclease (New England Biolabs, Beverly, MA, USA) at $37^{\circ} \mathrm{C}$ for $16 \mathrm{~h}$ and detected using $3.5 \%$ agarose gel electrophoresis. The primer sequences used to detect the miR-130a rs731384C >T polymorphism were 5'-GAT GCT CAG TCC TCA AAG AAC A-3' (forward) and 5'-TGA GGC CTA GAG CTC TGC TTT AT-3' (reverse). DNA was amplified over 35 cycles (denaturation at $95^{\circ} \mathrm{C}$ for $30 \mathrm{sec}$, annealing at $58^{\circ} \mathrm{C}$ for $30 \mathrm{sec}$, and extension at $72^{\circ} \mathrm{C}$ for $35 \mathrm{sec}$ ). The PCR products were digested with NlaIII (New England Biolabs) at $37^{\circ} \mathrm{C}$ for $16 \mathrm{~h}$ and were visualized using $3 \%$ agarose gel electrophoresis. The primer sequences used to detect the $m i R-150$ rs73056059G $>\mathrm{A}$ were 5'-GTT CCT GCC AGA GGA AGT G-3' (forward) and 5'-CCT CTG GAG TCC ACA CTC CAT-3' (reverse). DNA was amplified over 35 cycles (denaturation at $95^{\circ} \mathrm{C}$ for $30 \mathrm{sec}$, annealing at $60^{\circ} \mathrm{C}$ for $35 \mathrm{sec}$, and extension at $72^{\circ} \mathrm{C}$ for $40 \mathrm{sec}$ ). The products were digested with $\mathrm{BccI}$ (New England Biolabs) at $37^{\circ} \mathrm{C}$ for $16 \mathrm{~h}$ and detected using $4 \%$ gel electrophoresis. The primer sequences used to detect the $m i R-155$ rs767649T $>\mathrm{A}$ polymorphism were 5'-CCT GTA TGA CAA GGT TGT GTT TG-3' (forward) and 5'-GCT GGC ATA CTA TTC TAC CCA TAA-3' (reverse). DNA was amplified over 35 cycles (denaturation at $95^{\circ} \mathrm{C}$ for $35 \mathrm{sec}$, annealing at $56^{\circ} \mathrm{C}$ for $30 \mathrm{sec}$, and extension at $72^{\circ} \mathrm{C}$ for $\left.35 \mathrm{sec}\right)$. The PCR products were digested with Tsp45I (New England Biolabs) at $37^{\circ} \mathrm{C}$ for $16 \mathrm{~h}$ and visualized using $3 \%$ agarose gel electrophoresis.

Statistical analysis. Clinical characteristics were compared using the Student's unpaired t-test. Associations among ischemic stroke and the 4 miRNA genotypes were estimated by calculating the odd ratios (ORs) and 95\% confidence intervals (CIs) using the Fisher's exact test. Adjusted ORs (AORs) for the miRNA polymorphisms were determined using multiple logistic regression analysis based on gender, age, diabetes mellitus, hypertension, hyperlipidemia and smoking. The genotype distribution for each polymorphism was assessed for Hardy-Weinberg equilib- 
Table I. Baseline characteristics between patients with ischemic stroke and the control subjects.

\begin{tabular}{|c|c|c|c|}
\hline Characteristic & Controls $(n=404)$ & Stroke patients $(\mathrm{n}=596)$ & P-value \\
\hline Male (\%) & $173(42.8)$ & $254(42.6)$ & 0.953 \\
\hline Age (years, mean $\pm S D)$ & $63.66 \pm 10.47$ & $63.67 \pm 10.42$ & 0.987 \\
\hline Smoking (\%) & $138(34.2)$ & $211(35.4)$ & 0.704 \\
\hline Hypertension (\%) & $169(41.8)$ & $376(63.1)$ & 0.0002 \\
\hline Diabetes mellitus (\%) & $54(13.4)$ & $161(27.0)$ & $<0.0001$ \\
\hline Hyperlipidemia (\%) & $95(23.5)$ & $179(30.0)$ & 0.070 \\
\hline Homocysteine $(\mu \mathrm{mol} / 1$, mean $\pm \mathrm{SD})$ & $10.12 \pm 4.21$ & $11.26 \pm 6.78$ & 0.003 \\
\hline Folate $(\mathrm{nmol} / \mathrm{l}$, mean $\pm \mathrm{SD})$ & $8.88 \pm 7.99$ & $7.06 \pm 5.29$ & $<0.0001$ \\
\hline Vitamin $B_{12}(p g / m l$, mean $\pm S D)$ & $746.18 \pm 670.06$ & $747.46 \pm 624.35$ & 0.976 \\
\hline Total cholesterol $(\mathrm{mg} / \mathrm{dl}$, mean $\pm \mathrm{SD})$ & $193.70 \pm 37.59$ & $189.88 \pm 40.79$ & 0.137 \\
\hline Triglyceride $(\mathrm{mg} / \mathrm{dl}$, mean $\pm \mathrm{SD})$ & $147.74 \pm 90.39$ & $152.58 \pm 114.39$ & 0.480 \\
\hline $\operatorname{PLT}\left(10^{3} / \mathrm{ml}\right.$, mean $\left.\pm \mathrm{SD}\right)$ & $243.06 \pm 67.51$ & $248.19 \pm 87.73$ & 0.324 \\
\hline $\mathrm{PT}(\mathrm{sec}$, mean $\pm \mathrm{SD})$ & $11.77 \pm 0.79$ & $11.91 \pm 3.08$ & 0.440 \\
\hline aPTT $(\sec$, mean \pm SD $)$ & $33.34 \pm 18.61$ & $30.46 \pm 4.36$ & 0.001 \\
\hline Fibrinogen $(\mathrm{mg} / \mathrm{dl}$, mean $\pm \mathrm{SD})$ & $397.83 \pm 119.62$ & $426.93 \pm 132.40$ & 0.018 \\
\hline Antithrombin $(\%$, mean \pm SD $)$ & $94.36 \pm 43.88$ & $93.36 \pm 18.67$ & 0.685 \\
\hline BUN $(\mathrm{mg} / \mathrm{dl}$, mean $\pm \mathrm{SD})$ & $15.93 \pm 5.02$ & $16.29 \pm 7.80$ & 0.419 \\
\hline Uric acid (mg/dl, mean \pm SD) & $4.68 \pm 1.47$ & $4.69 \pm 1.67$ & 0.923 \\
\hline
\end{tabular}

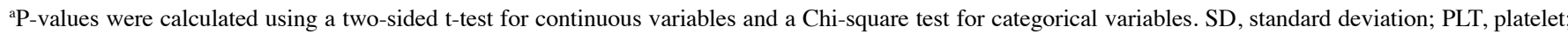
PT, prothrombin time; aPTT, activated partial thromboplastin time; BUN, blood urea nitrogen.

rium deviations and genotype and allele frequency differences between groups were assessed using $\chi^{2}$ tests. A value of $\mathrm{P}<0.05$ was considered to indicate a statistically significant difference. Stratification analysis was used to distinguish stroke subgroups based on the size of the occluded vessel. One-way analysis of variance (ANOVA) was performed to compare the mean homocysteine concentration levels among different genotypes. Stats Direct Statistical Software (version 2.4.4; StatsDirect Ltd., Altrincham, UK) was used to calculate the adjusted OR and 95\% CI. Survival curves were created using Cox proportional hazards regression, and the significance of differences between groups was assessed using the log-rank test. Cox regression models were used to analyze the independent prognostic importance of various markers, and results were adjusted for age, gender, diabetes mellitus, hypertension, hyperlipidemia and smoking. Hazard ratios (HRs) are presented with the 95\% CI. We also performed in silico analysis to identify potential transcription factor binding sites in miRNA promoter regions using Alibaba 2.1 (Grabe 2002), an online bio-informatics tool.

\section{Results}

The demographic characteristics and clinical variables of the ischemic stroke and control groups are shown in Table I. When comparing the ischemic stroke group with the control group, the stroke patients had a significantly higher incidence of hypertension and diabetes, significantly higher homocysteine and fibrinogen levels, and significantly lower folate levels and aPTT (all P<0.05) (Table I).

We investigated 4 miRNA polymorphisms, $m i R-34 a$ rs $6577555 \mathrm{C}>\mathrm{A}, \quad m i R-130 a$ rs $731384 \mathrm{C}>\mathrm{T}, \quad m i R-150$ rs73056059G $>\mathrm{A}$ and $m i R-155$ rs767649T $>\mathrm{A}$, and their associations with ischemic stroke. All the observed polymorphism frequencies were consistent with the Hardy-Weinberg equilibrium. However, we could not find any polymorphism frequency differences between the ischemic stroke and control groups (Table II). Once the ischemic stroke group was stratified into subgroups and analyzed, we observed associations between miRNA polymorphisms and individual stroke subtypes. LAD was significantly associated with the $m i R$-150GA genotype (GG vs. AA: AOR, 1.922; 95\% CI, 1.003-3.681). CE was also significantly associated with $m i R-150$ (GG vs. AA: AOR, 2.996; 95\% CI, 1.293-6.939) (these values are shown in bold in Table II). However, these differences dissipated once the P-value was adjusted for false discovery rate (FDR) (Table II). The $m i R-34 a \mathrm{C}>\mathrm{A}, m i R-130 a \mathrm{C}>\mathrm{T}$, and $m i R-155 \mathrm{~T}>\mathrm{A}$ polymorphisms did not differ significantly between the stroke patients and the control subjects.

We performed stratified analyses according to age, gender, hypertension, diabetes mellitus, hyperlipidemia, smoking, folate levels and homocysteine levels. The $m i R-34 a \mathrm{CA}+\mathrm{AA}$ genotype exhibited elevated prevalence in subjects who were $\geq 63$ years of age (AOR, 1.443; 95\% CI, 1.010-2.062), of the female gender (AOR, 1.459; 95\% CI, 1.026-2.076) and who were non-diabetic (AOR, 1.360; 95\% CI, 1.013-1.827). The miR-150GA+AA genotype also exhibited elevated prevalence in patients with hyperlipidemia (AOR, 6.060; 95\% CI, 1.358-27.04) (Table III).

Combined gene-environment analyses revealed several genotypes that were associated with clinical factors related to the risk of ischemic stroke. The miR-34aCA+AA genotype exhibited elevated stroke prevalence in subjects with hypertension (AOR, 3.088; 95\% CI, 2.089-4.566), hyperlipidemia (AOR, 1.570; 95\% CI, 1.010-2.443), smokers (AOR, 1.651; $95 \%$ CI, 1.023-2.665) and those with high homocysteine levels 


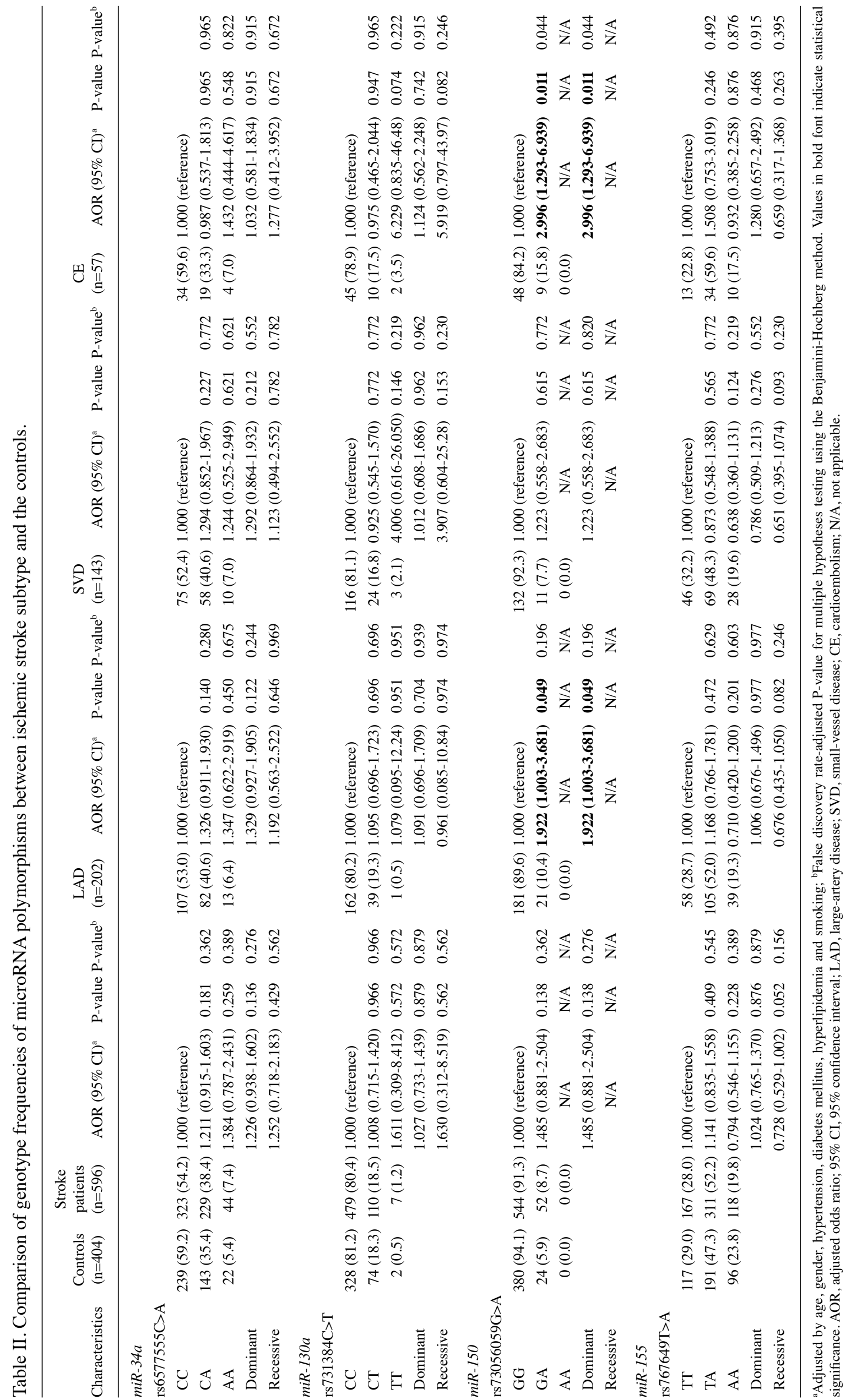




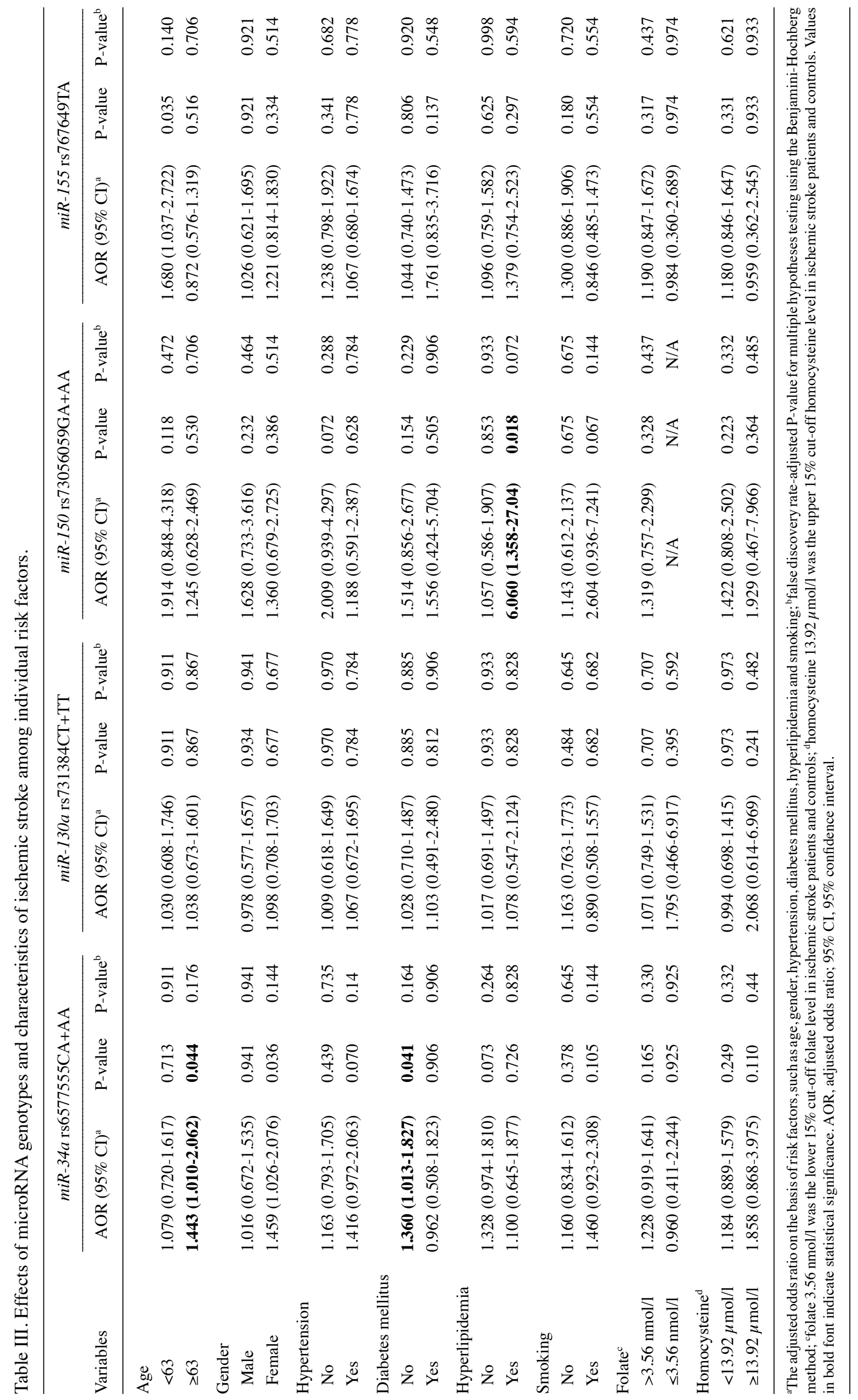




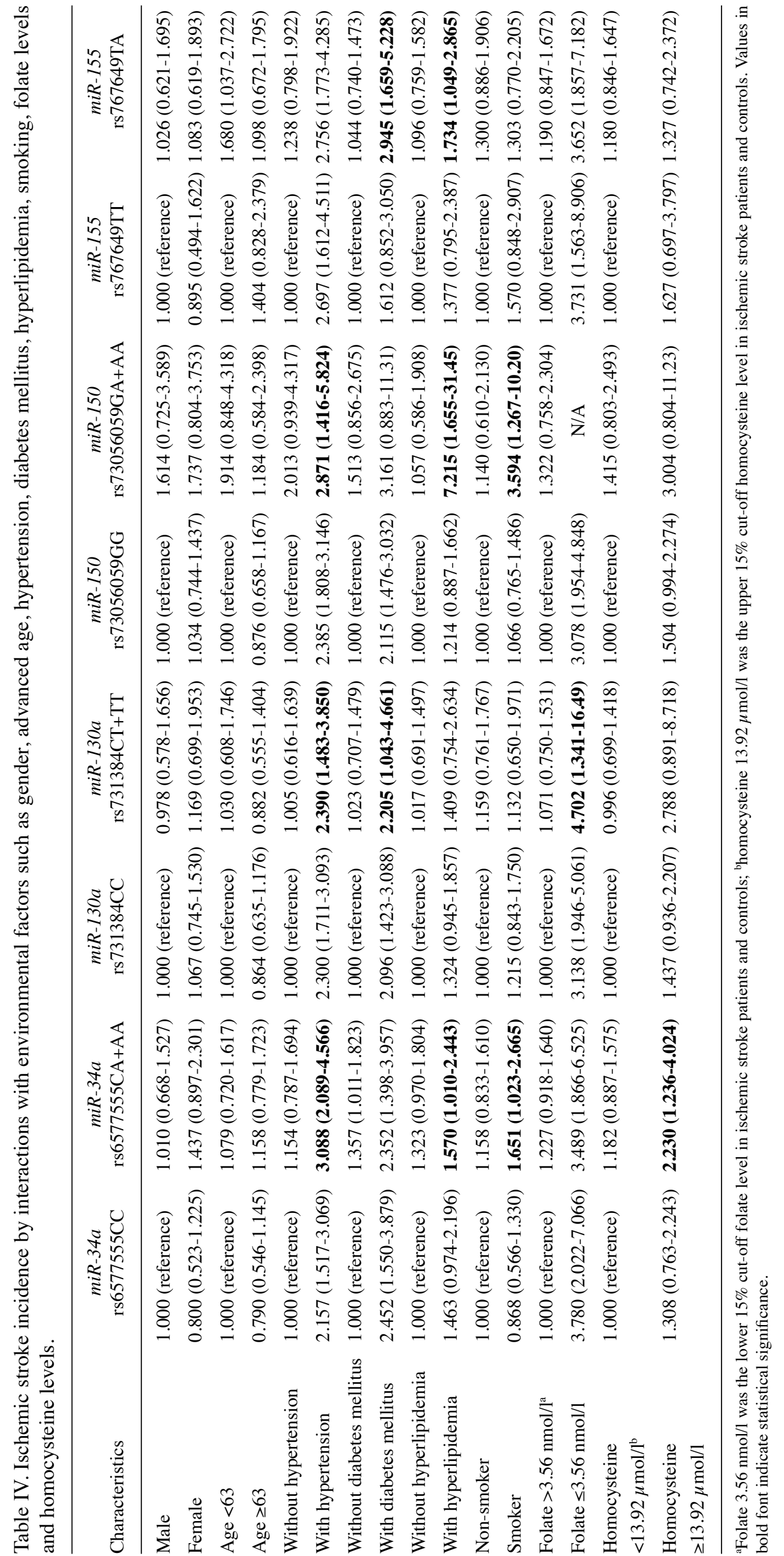


Table V. Allele combination analysis for the microRNA polymorphisms in patients with ischemic stroke and controls by MDR.

\begin{tabular}{|c|c|c|c|c|c|}
\hline Characteristics & $\begin{array}{c}\text { Overall } \\
(2 n=2000)\end{array}$ & $\begin{array}{l}\text { Controls } \\
(2 n=808)\end{array}$ & $\begin{array}{l}\text { Stroke patients } \\
\quad(2 \mathrm{n}=1192)\end{array}$ & OR $(95 \% \mathrm{CI})$ & P-value ${ }^{a}$ \\
\hline \multicolumn{6}{|c|}{$m i R-34 a \mathrm{C}>\mathrm{A} / m i R-130 a \mathrm{C}>\mathrm{T} / m i R-150 \mathrm{G}>\mathrm{A} / m i R-155 \mathrm{~T}>\mathrm{A}$} \\
\hline C-C-G-T & 0.341 & 0.341 & 0.344 & 1.000 (reference) & \\
\hline C-C-G-A & 0.308 & 0.330 & 0.292 & $0.878(0.704-1.095)$ & 0.260 \\
\hline C-C-A-T & 0.013 & 0.014 & 0.008 & $0.610(0.255-1.456)$ & 0.268 \\
\hline C-C-A-A & 0.012 & 0.011 & 0.015 & $1.341(0.594-3.030)$ & 0.552 \\
\hline C-T-G-T & 0.037 & 0.042 & 0.033 & $0.789(0.487-1.278)$ & 0.384 \\
\hline C-T-G-A & 0.033 & 0.030 & 0.036 & $1.202(0.713-2.026)$ & 0.516 \\
\hline C-T-A-T & 0.003 & 0.001 & 0.004 & $3.354(0.390-28.88)$ & 0.410 \\
\hline C-T-A-A & 0.001 & 0.000 & 0.002 & $3.356(0.160-70.22)$ & 0.519 \\
\hline A-C-G-T & 0.119 & 0.113 & 0.119 & $1.047(0.772-1.419)$ & 0.816 \\
\hline A-C-G-A & 0.098 & 0.092 & 0.104 & $1.124(0.811-1.557)$ & 0.510 \\
\hline A-C-A-T & 0.000 & 0.000 & 0.005 & $8.725(0.489-155.6)$ & 0.086 \\
\hline A-C-A-A & 0.008 & 0.004 & 0.009 & $2.236(0.610-8.200)$ & 0.263 \\
\hline A-T-G-T & 0.022 & 0.016 & 0.028 & $1.703(0.880-3.294)$ & 0.121 \\
\hline A-T-G-A & 0.005 & 0.008 & 0.000 & $0.052(0.003-0.921)$ & 0.004 \\
\hline A-T-A-A & 0.000 & 0.000 & 0.002 & $3.356(0.160-70.22)$ & 0.519 \\
\hline \multicolumn{6}{|c|}{$m i R-34 a \mathrm{C}>\mathrm{A} / m i R-130 a \mathrm{C}>\mathrm{T} / m i R-150 \mathrm{G}>\mathrm{A}$} \\
\hline C-C-G & 0.650 & 0.670 & 0.637 & 1.000 (reference) & \\
\hline C-C-A & 0.024 & 0.025 & 0.023 & $0.964(0.535-1.737)$ & 1.000 \\
\hline C-T-G & 0.070 & 0.072 & 0.069 & $1.010(0.709-1.438)$ & 1.000 \\
\hline C-T-A & 0.004 & 0.001 & 0.006 & $4.999(0.613-40.77)$ & 0.150 \\
\hline A-C-G & 0.216 & 0.205 & 0.222 & $1.143(0.914-1.429)$ & 0.258 \\
\hline A-C-A & 0.010 & 0.004 & 0.015 & $4.285(1.255-14.62)$ & 0.013 \\
\hline A-T-G & 0.027 & 0.023 & 0.029 & $1.315(0.744-2.325)$ & 0.399 \\
\hline \multicolumn{6}{|c|}{$m i R-34 a \mathrm{C}>\mathrm{A} / m i R-150 \mathrm{G}>\mathrm{A}$} \\
\hline $\mathrm{C}-\mathrm{G}$ & 0.719 & 0.743 & 0.704 & 1.000 (reference) & \\
\hline C-A & 0.029 & 0.026 & 0.030 & $1.226(0.708-2.122)$ & 0.496 \\
\hline$A-G$ & 0.243 & 0.228 & 0.252 & $1.170(0.947-1.445)$ & 0.149 \\
\hline A-A & 0.009 & 0.004 & 0.014 & $3.814(1.106-13.15)$ & 0.032 \\
\hline \multicolumn{6}{|c|}{$m i R-150 \mathrm{G}>\mathrm{A} / m i R-155 \mathrm{~T}>\mathrm{A}$} \\
\hline G-T & 0.520 & 0.512 & 0.528 & 1.000 (reference) & \\
\hline G-A & 0.443 & 0.459 & 0.429 & $0.904(0.754-1.085)$ & 0.285 \\
\hline A-T & 0.016 & 0.014 & 0.014 & $0.876(0.410-1.870)$ & 0.845 \\
\hline A-A & 0.023 & 0.015 & 0.030 & $1.970(1.013-3.831)$ & 0.049 \\
\hline
\end{tabular}

95\% CI, 95\% confidence interval; OR, odd ratios; ${ }^{a}$ Fisher's exact test. MDR, multifactor dimensionality reduction. Values in bold font indicate statistical significance.

(AOR, 2.230; 95\% CI, 1.236-4.024). The miR-130aCT+TT genotype also exhibited elevated stroke prevalence in subjects with hypertension (AOR, 2.390; 95\% CI, 1.4833.850), diabetes mellitus (AOR, 2.205; 95\% CI, 1.043-4.661) and low folate levels (AOR, 4.702; 95\% CI, 1.341-16.49). The $m i R-150 \mathrm{GA}+\mathrm{AA}$ genotype exhibited elevated stroke prevalence in subjects with hypertension (AOR, 2.871; 95\% CI, 1.416-5.824), hyperlipidemia (AOR, 7.215; 95\% CI, 1.655-31.45) and who were smokers (AOR, 3.594; 95\% CI, 1.267-10.20). The miR-155TA genotype exhibited elevated stroke prevalence in subjects with diabetes mellitus (AOR, 2.945; 95\% CI, 1.659-5.228) and hyperlipidemia (AOR, 1.734; 95\% CI, 1.049-2.865) (Table IV).
We then performed allelic combination analyses using the multifactor dimensionality reduction method comparing the ischemic stroke patients and the control subjects (Table V). The following allele combinations exhibited a significant association with the prevalence of stroke $(\mathrm{P}>0.05)$ : the A-T-G-A allele combination of $m i R-34 a \mathrm{C}>\mathrm{A} /$ $m i R-130 a \mathrm{C}>\mathrm{T} / m i R-150 \mathrm{G}>\mathrm{A} / m i R-155 \mathrm{~T}>\mathrm{A}(\mathrm{OR}, 0.052 ; 95 \% \mathrm{CI}$, 0.003-0.921), the A-C-A allele combination of $m i R-34 a \mathrm{C}>\mathrm{A} /$ $m i R-130 a \mathrm{C}>\mathrm{T} / m i R-150 \mathrm{G}>\mathrm{A}(\mathrm{OR}, 4.285 ; 95 \% \mathrm{CI}, 1.255-14.62)$, the A-A allele combination of $m i R-34 a \mathrm{C}>\mathrm{A} / m i R-150 \mathrm{G}>\mathrm{A}(\mathrm{OR}$, 3.814; 95\% CI, 1.106-13.15), and the A-A allele combination of $m i R-150 \mathrm{G}>\mathrm{A} / m i R-155 \mathrm{~T}>\mathrm{A}(\mathrm{OR}, 1.970 ; 95 \% \mathrm{CI}, 1.013-3.831)$. We also performed genotype combination analyses. The 
Table VI. Combined genotype analysis for the microRNA polymorphisms in patients with ischemic stroke and controls.

\begin{tabular}{|c|c|c|c|c|}
\hline Genotype & $\begin{array}{l}\text { Controls } \\
(\mathrm{n}=404)\end{array}$ & $\begin{array}{l}\text { Stroke patients } \\
\quad(\mathrm{n}=596)\end{array}$ & $\operatorname{AOR}(95 \% \mathrm{CI})^{\mathrm{a}}$ & $\mathrm{P}$-value ${ }^{\mathrm{b}}$ \\
\hline \multicolumn{5}{|c|}{$m i R-34 a \mathrm{C}>\mathrm{A} / m i R-130 a \mathrm{C}>\mathrm{T}$} \\
\hline $\mathrm{CC}-\mathrm{CC}$ & $193(47.8)$ & $260(43.6)$ & 1.000 (reference) & \\
\hline CC-CT & $45(11.1)$ & $57(9.6)$ & $0.946(0.601-1.488)$ & 0.809 \\
\hline CC-TT & $1(0.2)$ & $6(1.0)$ & $4.979(0.566-43.81)$ & 0.148 \\
\hline $\mathrm{CA}-\mathrm{CC}$ & $119(29.5)$ & $187(31.4)$ & $1.231(0.904-1.675)$ & 0.187 \\
\hline CA-CT & $23(5.7)$ & $41(6.9)$ & $1.245(0.700-2.214)$ & 0.456 \\
\hline CA-TT & $1(0.2)$ & $1(0.2)$ & $0.816(0.050-13.29)$ & 0.887 \\
\hline AA-CC & $16(4.0)$ & $32(5.4)$ & $1.405(0.721-2.740)$ & 0.318 \\
\hline AA-CT & $6(1.5)$ & $12(2.0)$ & $2.201(0.752-6.445)$ & 0.150 \\
\hline \multicolumn{5}{|c|}{$m i R-34 a \mathrm{C}>\mathrm{A} / m i R-150 \mathrm{G}>\mathrm{A}$} \\
\hline CC-GG & $220(54.5)$ & $297(49.8)$ & 1.000 (reference) & \\
\hline CC-GA & $19(4.7)$ & $26(4.4)$ & $1.036(0.543-1.977)$ & 0.915 \\
\hline CA-GG & $140(34.7)$ & 207 (34.7) & $1.134(0.851-1.513)$ & 0.391 \\
\hline CA-GA & $3(0.7)$ & $22(3.7)$ & $5.470(1.580-18.93)$ & 0.007 \\
\hline AA-GG & $20(5.0)$ & $40(6.7)$ & $1.570(0.869-2.836)$ & 0.135 \\
\hline AA-GA & $2(0.5)$ & $4(0.7)$ & $1.329(0.220-8.028)$ & 0.757 \\
\hline \multicolumn{5}{|c|}{$m i R-34 a \mathrm{C}>\mathrm{A} / m i R-155 \mathrm{~T}>\mathrm{A}$} \\
\hline CC-TT & $64(15.8)$ & $90(15.1)$ & 1.000 (reference) & \\
\hline CC-TA & $115(28.5)$ & $166(27.9)$ & $1.081(0.706-1.653)$ & 0.721 \\
\hline CC-AA & $60(14.9)$ & $67(11.2)$ & $0.834(0.508-1.367)$ & 0.471 \\
\hline CA-TT & $49(12.1)$ & $63(10.6)$ & $1.055(0.624-1.784)$ & 0.841 \\
\hline CA-TA & $65(16.1)$ & $120(20.1)$ & $1.428(0.891-2.287)$ & 0.139 \\
\hline CA-AA & $29(7.2)$ & $46(7.7)$ & $1.020(0.561-1.855)$ & 0.949 \\
\hline AA-TT & $4(1.0)$ & $14(2.3)$ & $3.351(0.965-11.64)$ & 0.057 \\
\hline AA-TA & $11(2.7)$ & $25(4.2)$ & $1.892(0.820-4.366)$ & 0.135 \\
\hline AA-AA & $7(1.7)$ & $5(0.8)$ & $0.347(0.091-1.317)$ & 0.120 \\
\hline \multicolumn{5}{|c|}{$m i R-130 a \mathrm{C}>\mathrm{T} / m i R-150 \mathrm{G}>\mathrm{A}$} \\
\hline CC-GG & $307(76.0)$ & $438(73.5)$ & 1.000 (reference) & \\
\hline CC-GA & $21(5.2)$ & $41(6.9)$ & $1.308(0.742-2.306)$ & 0.354 \\
\hline CT-GG & $71(17.6)$ & $101(16.9)$ & $0.965(0.679-1.371)$ & 0.841 \\
\hline CT-GA & $3(0.7)$ & $9(1.5)$ & $2.292(0.590-8.897)$ & 0.231 \\
\hline TT-GG & $2(0.5)$ & $5(0.8)$ & $1.660(0.311-8.879)$ & 0.553 \\
\hline \multicolumn{5}{|c|}{$m i R-130 a \mathrm{C}>\mathrm{T} / m i R-155 \mathrm{~T}>\mathrm{A}$} \\
\hline CC-TT & $91(22.5)$ & $135(22.7)$ & 1.000 (reference) & \\
\hline CC-TA & $156(38.6)$ & $243(40.8)$ & $1.057(0.745-1.499)$ & 0.757 \\
\hline CC-AA & $81(20.0)$ & $101(16.9)$ & $0.747(0.493-1.131)$ & 0.168 \\
\hline CT-TT & $26(6.4)$ & $29(4.9)$ & $0.718(0.384-1.344)$ & 0.301 \\
\hline CT-TA & $33(8.2)$ & $64(10.7)$ & $1.217(0.718-2.062)$ & 0.465 \\
\hline CT-AA & $15(3.7)$ & $17(2.9)$ & $0.747(0.345-1.618)$ & 0.459 \\
\hline TT-TA & $2(0.5)$ & $4(0.7)$ & $1.395(0.245-7.949)$ & 0.708 \\
\hline \multicolumn{5}{|c|}{$m i R-150 \mathrm{G}>\mathrm{A} / m i R-155 \mathrm{~T}>\mathrm{A}$} \\
\hline GG-TT & $109(27.0)$ & $161(27.0)$ & 1.000 (reference) & \\
\hline GG-TA & $183(45.3)$ & $273(45.8)$ & $1.008(0.730-1.392)$ & 0.962 \\
\hline GG-AA & $88(21.8)$ & $110(18.5)$ & $0.796(0.540-1.173)$ & 0.248 \\
\hline GA-TT & $8(2.0)$ & $6(1.0)$ & $0.570(0.181-1.799)$ & 0.338 \\
\hline GA-TA & $8(2.0)$ & $38(6.4)$ & $3.265(1.426-7.474)$ & 0.005 \\
\hline GA-AA & $8(2.0)$ & $8(1.3)$ & $0.519(0.176-1.534)$ & 0.236 \\
\hline
\end{tabular}

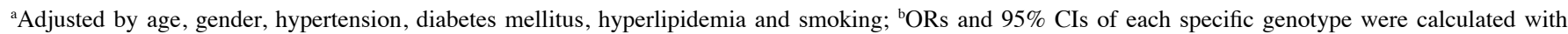
reference to frequencies of all others. Values in bold font indicate statistical significance. CI, confidence interval; OR, odd ratios. 


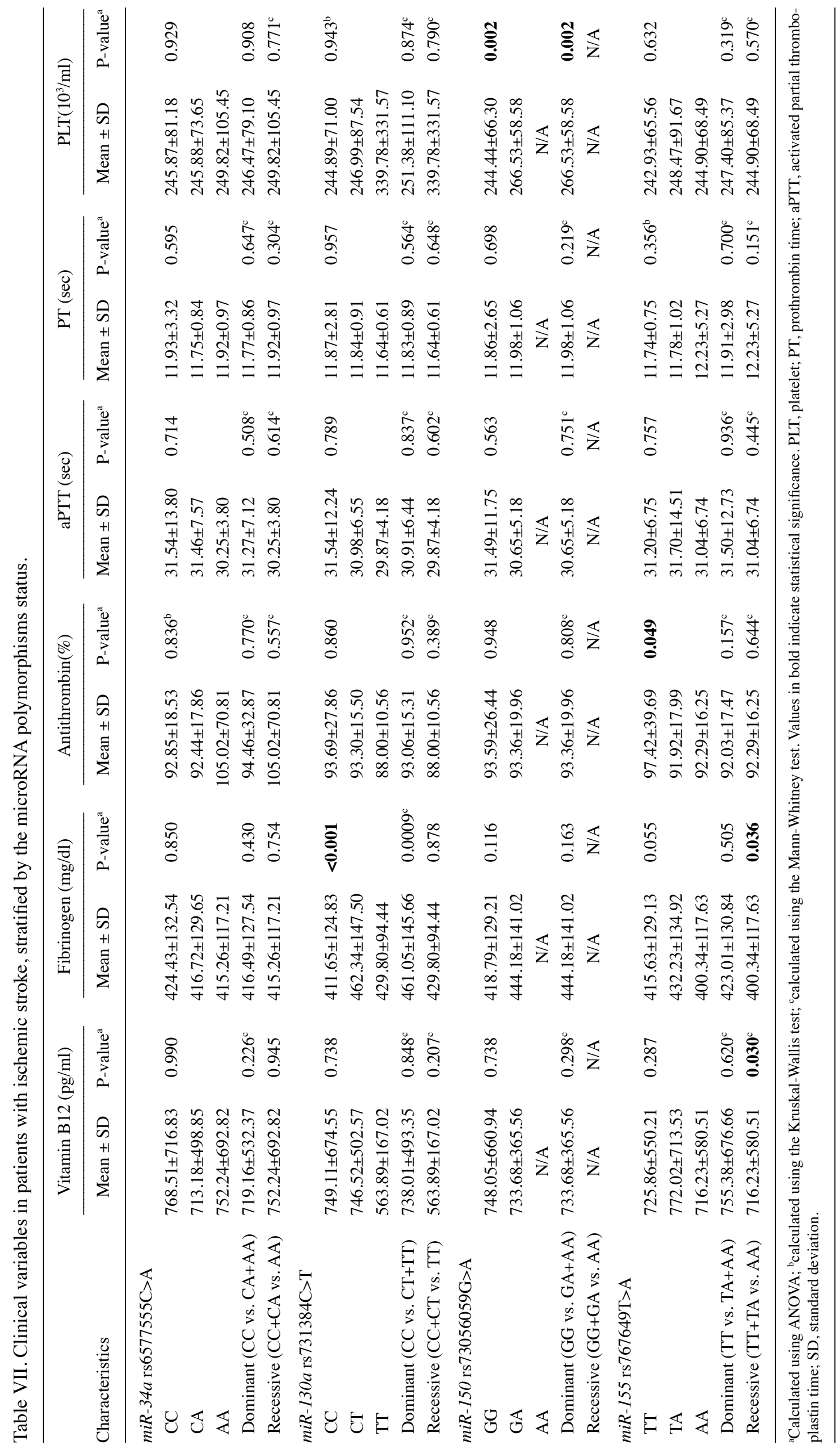



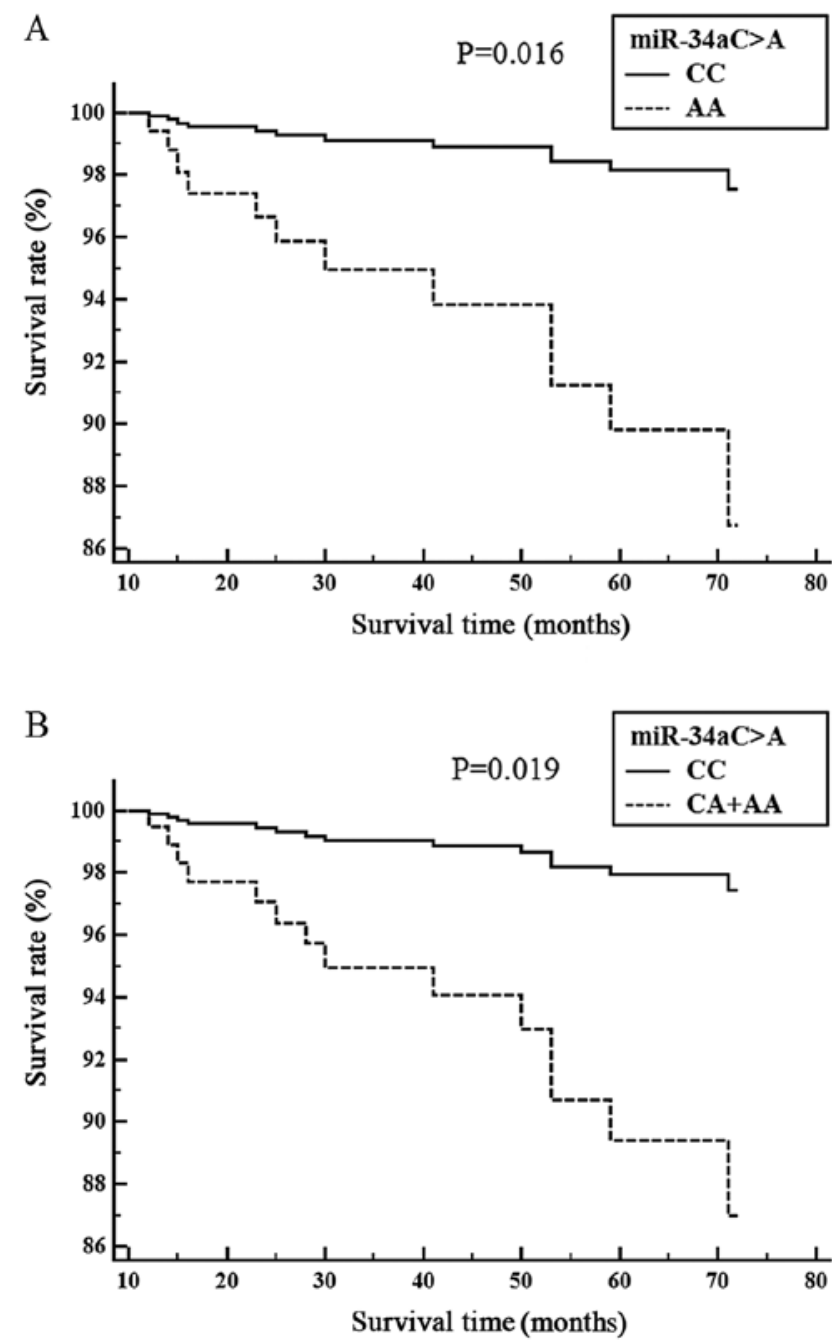

Figure 1. Cox proportional hazards regression of survival of patients with smallvessel disease (SVD) according to $m i R-34 a \mathrm{C}>\mathrm{A}$ polymorphisms. (A) Survival curves for the $m i R-34 a \mathrm{C}>\mathrm{A}$ polymorphism (CC vs. $\mathrm{CA})(\mathrm{P}=0.016)$. (B) Survival curves for the $m i R-34 a \mathrm{C}>\mathrm{A}$ polymorphism (CC vs. $\mathrm{CA}+\mathrm{AA})(\mathrm{P}=0.019)$.

$m i R-34 a \mathrm{CA} / m i R-150 \mathrm{GA}$ genotype (AOR, 5.470; 95\% CI, $1.580-18.932)$ and the $m i R-150 \mathrm{GA} / m i R-155 \mathrm{TA}$ genotype (AOR, 3.265; 95\% CI, 1.426-7.474) were both associated with an increased prevalence of ischemic stroke (Table VI).

The miR-130a genotype was associated with fibrinogen levels (CC vs. CT vs. TT: CC, $411.65 \pm 124.83$;

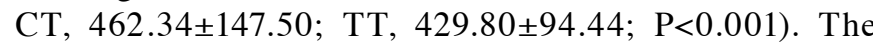
miR-150 dominant model (GG vs. GA+AA) was significantly associated with increased platelet counts (GG vs. GA+AA: GG, 244.44 $\pm 66.30 ; \mathrm{GA}+\mathrm{AA}, 266.53 \pm 58.58 ; \mathrm{P}=0.002)$. The $m i R-155$ recessive model (TT+TA vs. AA) was associated with vitamin B12 (TT+TA vs. AA: TT+TA, 748.94 \pm 631.87 ; AA, $716.23 \pm 580.51 ; \mathrm{P}=0.030)$ and fibrinogen levels (TT+TA vs. AA: TT+TA, 423.93 \pm 177.56 ; AA, 400.34 $\pm 117.63 ; \mathrm{P}=0.036$ ), and the miR-155 genotype was associated with antithrombin levels (TT vs. TA vs. AA: TT, 97.42 \pm 39.69 ; TA, 91.92 \pm 17.99 ; AA, 92.29 $\pm 16.25 ; \mathrm{P}=0.049$ ) (Table VII).

The association between the miRNA polymorphisms and ischemic stroke patient survival is shown (Figs. 1 and 2). Cox proportional analysis indicated that the $m i R-34 a \mathrm{CA}$ genotype was significantly associated with survival in the SVD

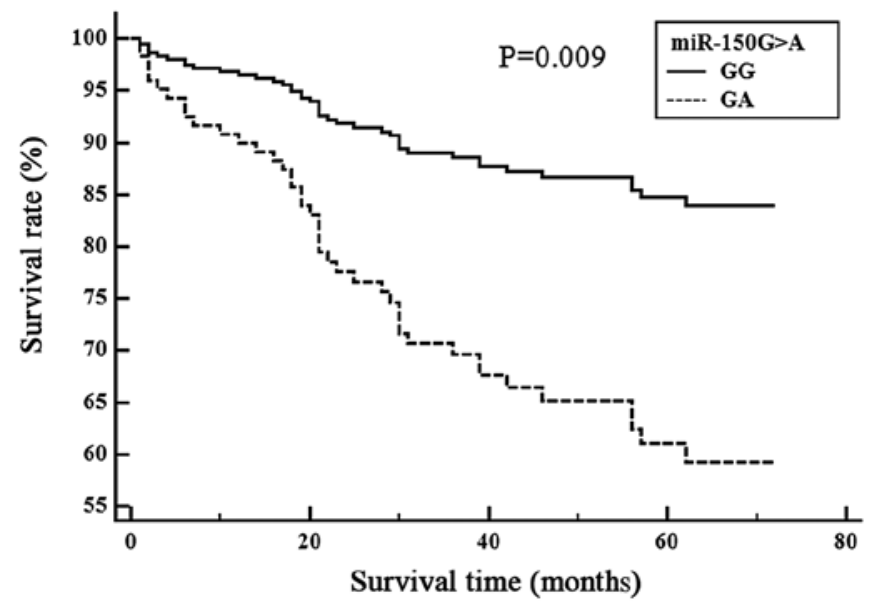

Figure 2. Cox proportional hazards regression of survival of patients with large-artery disease (LAD) according to $m i R-150 \mathrm{G}>\mathrm{A}$ polymorphisms. Survival curves for the $m i R-150 \mathrm{G}>\mathrm{A}$ polymorphism (GG vs. GA) $(\mathrm{P}=0.009)$.

subgroup (CC vs. $\mathrm{CA}, \mathrm{P}=0.016)$ (Fig. $1 \mathrm{~A})$, (CC vs. $\mathrm{CA}+\mathrm{AA}$, $\mathrm{P}=0.019$ ) (Fig. 1B). The miR-150GA genotype was associated with survival in the LAD subgroup (GG vs. GA, $\mathrm{P}=0.009$ ) (Fig. 2). We then investigated the promoter binding site for the miR-150 polymorphism.

\section{Discussion}

In this study, we selected a few potentially relevant miRNA SNPs, $m i R-34 a$ rs6577555C $>\mathrm{A}, m i R-130 a$ rs731384C $>\mathrm{T}$, $m i R-150$ rs73056059G $>\mathrm{A}$ and $m i R-155$ rs767649T $>\mathrm{A}$, and investigated their association with ischemic stroke in a Korean population. We found that the $m i R-150 \mathrm{G}>\mathrm{A}$ polymorphism may be associated with a susceptibility for LAD and CE ischemic stroke. In the gene-environment interaction analysis, the $m i R-150 \mathrm{GA}+\mathrm{AA}$ genotype combined with hyperlipidemia or smoking exhibited a significantly higher prevalence of ischemic stroke. In the allelic combination analysis, the A-A allele $(m i R-34 a \mathrm{C}>\mathrm{A}$ and $m i R-150 \mathrm{G}>\mathrm{A})$ significantly increased ischemic stroke prevalence overall. In particular, subjects with the miR-150A allele exhibited a marked increase in ischemic stroke incidence, whereas subjects with the $m i R-150 G$ allele had a decreased prevalence of ischemic stroke. These data were confirmed in combination analysis, where the $m i R-150 \mathrm{GA}$ genotype also exhibited an increased prevalence of ischemic stroke. The miR-150GA genotype was also associated with a high adjusted HR in the survival analysis in both the overall ischemic stroke patients and the LAD and CE subtypes. Additionally, subjects with the miR-150GA genotype had significantly higher platelet counts.

miRNAs play crucial roles in a number of physiological and pathological processes, including neurodegenerative diseases (25), epigenetics $(26,27)$, coronary artery disease (28), metabolism (14), tumorigenesis (29,30), angiogenesis and colonization (31). miRNAs play roles in oncogenesis, heart disease and nervous system function. It has been suggested that miRNAs may be used as biomarkers for cardiovascular diseases, including coronary artery disease, stroke, acute myocardial infarction and heart failure (17,32-34). 
$m i R-34 a$ regulates the myeloblastosis transcription factor MYB (35); miR-130a regulates V-maf avian musculoaponeurotic fibrosarcoma oncogene homolog B (MAFB) (36); $m i R-150$ regulates c-MYB (37); and $m i R-155$ regulates V-Ets avian erythroblastosis virus E26 oncogene homolog-1 (ETS-1) and Meis homeobox-1 (MEIS-1) (38). Previous studies have reported that these genes contribute to suppressing megakaryocytopoiesis and megakaryocyte differentiation $(36,39,40)$. MKs are platelet precursors, and excessive MK differentiation results in abnormal platelet production. Studies have been carried out investigating the causal association between increased platelets and ischemic stroke risk (41-43).

We then searched for transcription factors that could potentially bind the $m i R-150$ promoter near the $r 573056059 \mathrm{G}>\mathrm{A}$ nucleotide. For the A allele, we found an additional transcription binding site for LyF-1 and YY-1, which could alter $m i R$-150 levels (44). Transcription factor YY1 is associated with increased promoter activity (45-47); therefore, we hypothesize that $m i R-150$ expression is increased by YY1 in subjects with an A allele. Supporting this hypothesis, we performed an ANOVA, which indicated that the miR-150GA genotype had higher platelet counts than the GG type. However, we were unable to directly measure promoter activity for each miRNA polymorphisms in the present study.

This study has several limitations. First, it is not yet clear which genetic polymorphisms predict the ischemic stroke phenotypes. This study population included only Korean individuals, and our findings need to be validated in other ethnic groups. Second, the patient population included in the subgroup analyses was relatively small. Future studies would need to include $>1,000$ individuals from an ethnically homogeneous population. As Koreans have a low degree of interracial marriage, this should be sufficient to provide reliable data. Third, the controls included in our study were not subjected to stringent inclusion criteria, as the enrollment rate was relatively low compared to that of stroke patients. Therefore, we could not clearly identify the casual effects of vascular risk factors in these subjects. Lastly, our results cannot be extrapolated to other ethnic groups, as racial variations in the allelic frequency could produce different results.

In conclusion, in this study, we identified a significant association between the $m i R-150 \mathrm{G}>\mathrm{A}$ polymorphism and ischemic stroke in Korean individuals. This study demonstrates that the $m i R$-150GA genotype occurs at a higher frequency in stroke patients, which suggests that $m i R-150$ may play a role in the prevalence of ischemic stroke. Therefore, our findings suggest that $m i R-150$ polymorphisms may contribute to ischemic stroke and may potentially act as biomarkers for the diagnosis and the risk of ischemic stroke. To the best of our knowledge, this is the first study to evaluate the association between miRNA polymorphisms ( $m i R-34 a \mathrm{C}>\mathrm{A}, m i R-130 a \mathrm{C}>\mathrm{T}, m i R-150 \mathrm{G}>\mathrm{A}$ and $m i R-155 \mathrm{~T}>\mathrm{A}$ ) and ischemic stroke in a Korean population. Therefore, further studies on miRNA polymorphisms in other racial or ethnic populations are required in order to fully elucidate their role in the risk of ischemic stroke.

\section{Acknowledgements}

This study was supported by the National Research Foundation of Korea Grant funded by the Korean Government (NRF-2013R1A1A2008177 and NRF-2014R1A1A2059118).

\section{References}

1. Donnan GA, Fisher M, Macleod M and Davis SM: Stroke. Lancet 371: 1612-1623, 2008

2. Goldstein LB, Adams R, Becker K, Furberg CD, Gorelick PB, Hademenos G, Hill M, Howard G, Howard VJ, Jacobs B, et al: Primary prevention of ischemic stroke: A statement for healthcare professionals from the Stroke Council of the American Heart Association. Circulation 103: 163-182, 2001.

3. Mustacchi P: Risk factors in stroke. West J Med 143: 186-192, 1985.

4. Arboix A: Cardiovascular risk factors for acute stroke: Risk profiles in the different subtypes of ischemic stroke. World J Clin Cases 3: 418-429, 2015.

5. Mohr JP, Caplan LR, Melski JW, Goldstein RJ, Duncan GW, Kistler JP, Pessin MS and Bleich HL: The Harvard Cooperative Stroke Registry: A prospective registry. Neurology 28: 754-762, 1978.

6. Cosemans JM, Angelillo-Scherrer A, Mattheij NJ and Heemskerk JW: The effects of arterial flow on platelet activation, thrombus growth, and stabilization. Cardiovasc Res 99: 342-352, 2013.

7. Shah AB, Beamer N and Coull BM: Enhanced in vivo platelet activation in subtypes of ischemic stroke. Stroke 16: 643-647, 1985.

8. Sharma SC, Vijayan GP, Suri ML and Seth HN: Platelet adhesiveness in young patients with ischaemic stroke. J Clin Pathol 30: 649-652, 1977.

9. O'Malley T, Langhorne P, Elton RA and Stewart C: Platelet size in stroke patients. Stroke 26: 995-999, 1995.

10. George JN: Platelets. Lancet 355: 1531-1539, 2000.

11. Pease DC: An electron microscopic study of red bone marrow. Blood 11: 501-526, 1956.

12. Ambros V: The functions of animal microRNAs. Nature 431: 350-355, 2004

13. Bartel DP: MicroRNAs: Target recognition and regulatory functions. Cell 136: 215-233, 2009.

14. Aumiller V and Forstemann K: Roles of microR NAs beyond development - metabolism and neural plasticity. Biochim Biophys Acta 692-696: 2008, 1779.

15. Merkerova $\mathbf{M}$, Belickova $\mathbf{M}$ and Bruchova $\mathrm{H}$ : Differential expression of microRNAs in hematopoietic cell lineages. Eur J Haematol 81: 304-310, 2008.

16. Carissimi C, Fulci V and Macino G: MicroRNAs: Novel regulators of immunity. Autoimmun Rev 8: 520-524, 2009.

17. Tan KS, Armugam A, Sepramaniam S, Lim KY, Setyowati KD, Wang CW and Jeyaseelan K: Expression profile of MicroRNAs in young stroke patients. PLoS One 4: e7689, 2009.

18. Liu DZ, Tian Y, Ander BP, Xu H, Stamova BS, Zhan X, Turner RJ, Jickling $G$ and Sharp FR: Brain and blood microRNA expression profiling of ischemic stroke, intracerebral hemorrhage, and kainate seizures. J Cereb Blood Flow Metab 30: 92-101, 2010.

19. Jeon YJ, Kim OJ, Kim SY, Oh SH, Oh D, Kim OJ, Shin BS and Kim NK: Association of the miR-146a, miR-149, miR-196a2, and miR-499 polymorphisms with ischemic stroke and silent brain infarction risk. Arterioscler Thromb Vasc Biol 33: 420-430, 2013.

20. Edelstein LC and Bray PF: MicroRNAs in platelet production and activation. Blood 117: 5289-5296, 2011.

21. Dangwal S and Thum T: MicroRNAs in platelet biogenesis and function. Thromb Haemost 108: 599-604, 2012.

22. Goldstein LB, Jones MR, Matchar DB, Edwards LJ, Hoff J, Chilukuri V, Armstrong SB and Horner RD: Improving the reliability of stroke subgroup classification using the Trial of ORG 10172 in Acute Stroke Treatment (TOAST) criteria. Stroke 32: 1091-1098, 2001

23. Kim OJ, Hong SH, Oh SH, Kim TG, Min KT, Oh D and Kim NK: Association between VEGF polymorphisms and homocysteine levels in patients with ischemic stroke and silent brain infarction. Stroke 42: 2393-2402, 2011.

24. Jeon YJ, Kim YR, Lee BE, Choi YS, Kim JH, Shin JE, Rah H, Cha SH, Lee WS and Kim NK: Genetic association of five plasminogen activator inhibitor-1 (PAI-1) polymorphisms and idiopathic recurrent pregnancy loss in Korean women. Thromb Haemost 110: 742-750, 2013.

25. Bushati N and Cohen SM: MicroRNAs in neurodegeneration. Curr Opin Neurobiol 18: 292-296, 2008.

26. Chuang JC and Jones PA: Epigenetics and microRNAs. Pediatr Res 61: 24R-29R, 2007.

27. Saetrom P, Snøve O Jr and Rossi JJ: Epigenetics and microRNAs. Pediatr Res 61: 17R-23R, 2007. 
28. Gao H, Guddeti RR, Matsuzawa Y, Liu LP, Su LX, Guo D, Nie SP, Du J and Zhang M: Plasma levels of microRNA-145 are associated with severity of coronary artery disease. PLoS One 10: e0123477, 2015.

29. Lujambio A and Esteller M: CpG island hypermethylation of tumor suppressor microRNAs in human cancer. Cell Cycle 6: 1455-1459, 2007.

30. Lu J, Getz G, Miska EA, Alvarez-Saavedra E, Lamb J, Peck D, Sweet-Cordero A, Ebert BL, Mak RH, Ferrando AA, et al: MicroRNA expression profiles classify human cancers. Nature 435: 834-838, 2005

31. Zhang H, Li Y and Lai M: The microRNA network and tumor metastasis. Oncogene 29: 937-948, 2010.

32. Jeyaseelan K, Lim KY and Armugam A: MicroRNA expression in the blood and brain of rats subjected to transient focal ischemia by middle cerebral artery occlusion. Stroke 39: 959-966, 2008.

33. Fichtlscherer S, De Rosa S, Fox H, Schwietz T, Fischer A, Liebetrau C, Weber M, Hamm CW, Röxe T, Müller-Ardogan M, et al: Circulating microRNAs in patients with coronary artery disease. Circ Res 107: 677-684, 2010.

34. Laterza OF, Lim L, Garrett-Engele PW, Vlasakova K, Muniappa N, Tanaka WK, Johnson JM, Sina JF, Fare TL, Sistare FD and Glaab WE: Plasma microRNAs as sensitive and specific biomarkers of tissue injury. Clin Chem 55: 1977-1983, 2009.

35. Navarro F, Gutman D, Meire E, Cáceres M, Rigoutsos I, Bentwich Z and Lieberman J: miR-34a contributes to megakaryocytic differentiation of K562 cells independently of p53. Blood 114: 2181-2192, 2009.

36. Garzon R, Pichiorri F, Palumbo T, Iuliano R, Cimmino A, Aqeilan R, Volinia S, Bhatt D, Alder H, Marcucci G, et al: MicroRNA fingerprints during human megakaryocytopoiesis. Proc Natl Acad Sci USA 103: 5078-5083, 2006.

37. Barroga CF, Pham $\mathrm{H}$ and Kaushansky K: Thrombopoietin regulates $\mathrm{c}-\mathrm{Myb}$ expression by modulating micro RNA 150 expression. Exp Hematol 36: 1585-1592, 2008.
38. Georgantas RW III, Hildreth R, Morisot S, Alder J, Liu CG, Heimfeld S, Calin GA, Croce CM and Civin CI: CD34+ hematopoietic stem-progenitor cell microRNA expression and function: A circuit diagram of differentiation control. Proc Natl Acad Sci USA 104: 2750-2755, 2007.

39. Ramsay RG: c-Myb a stem-progenitor cell regulator in multiple tissue compartments. Growth Factors 23: 253-261, 2005.

40. Lu J, Guo S, Ebert BL, Zhang H, Peng X, Bosco J, Pretz J, Schlanger R, Wang JY, Mak RH, et al: MicroRNA-mediated control of cell fate in megakaryocyte-erythrocyte progenitors. Dev Cell 14: 843-853, 2008.

41. Barnett HJ: Platelet and coagulation function in relation to thromboembolic stroke. Adv Neurol 16: 45-70, 1977.

42. Kalendovsky Z, Austin J and Steele P: Increased platelet aggregability in young patients with stroke. Diagnosis and therapy. Arch Neurol 32: 13-20, 1975.

43. van Rooy MJ and Pretorius E: Metabolic syndrome, platelet activation and the development of transient ischemic attack or thromboembolic stroke. Thromb Res 135: 434-442, 2015.

44. Grabe N: AliBaba2: Context specific identification of transcription factor binding sites. In Silico Biol 2: S1-S15, 2002.

45. Momoeda M, Kawase M, Jane SM, Miyamura K, Young NS and Kajigaya S: The transcriptional regulator YY1 binds to the 5'-terminal region of B19 parvovirus and regulates P6 promoter activity. J Virol 68: 7159-7168, 1994.

46. Lee GR: Role of YY1 in long-range chromosomal interactions regulating Th2 cytokine expression. Transcription 5: e27976, 2014.

47. Funahashi N, Hirota Y, Nakagawa K, Sawada N, Watanabe M, Suhara Y and Okano T: YY1 positively regulates human UBIAD1 expression. Biochem Biophys Res Commun 460: 238-244, 2015. 